\title{
A Theory-Based Self-Management Training Program for Older Adult Peer Leaders with Diabetes: A Feasibility Assessment
}

This article was published in the following Dove Press journal: Journal of Multidisciplinary Healthcare

\author{
Wen-Chun Chen (1D ${ }^{1-3}$ \\ Chiu-Chu Lin $\mathbb{D}^{2,4,5}$ \\ Chia-Chi Kuo $\mathbb{D}^{6,7}$ \\ Chia-Chen $\mathrm{Wu}\left(\mathbb{D}^{8}{ }^{8}\right.$ \\ Tz-Jie Liu (iD) ${ }^{9}$ \\ Mei-Tsu Chen (D) ${ }^{9}$
}

'Department of Community Medicine, St. Martin De Porres Hospital, Chiayi,

Taiwan; ${ }^{2}$ School of Nursing, Kaohsiung Medical University, Kaohsiung, Taiwan;

${ }^{3}$ Department of Nursing, Chang Jung

University of Science and Technology,

Chiayi Campus, Tainan, Taiwan;

${ }^{4}$ Department of Renal Care, College of

Medicine, Kaohsiung Medical University,

Kaohsiung, Taiwan; ${ }^{5}$ Department of

Medical Research, Kaohsiung Medical

University Hospital, Kaohsiung Medical

University, Kaohsiung, Taiwan;

${ }^{6}$ Emergency Department, Chi-Mei

Medical Center, Tainan, Taiwan;

${ }^{7}$ Department of Nursing, Chang Jung

Christian University, Tainan, Taiwan;

${ }^{8}$ School of Nursing, Fooyin University,

Kaohsiung, Taiwan; ${ }^{9}$ Health Management

Center, St. Martin De Porres Hospital,

Chiayi, Taiwan
Correspondence: Chiu-Chu Lin

Email chiuchu@kmu.edu.tw
Objective: To improve the quality of peer leader training, this study developed a theorybased self-management training program for older adult peer leaders with diabetes and assessed its feasibility.

Background: Current self-management programs are designed mainly to be implemented by healthcare professionals, but healthcare staff may not fully perceive the needs and obstacles of older adults in disease management due to a lack of similar illness experience. To target this problem, peer leaders with successful self-management experiences, similar cultural backgrounds and languages, and related illness experiences are trained to guide and mentor peer patients in self-management programs.

Study Design and Methods: This study was conducted in two stages. In stage 1, a peer leader training program was developed based on experiential learning theory as the framework and self-regulation theory as the activity design strategy. In stage 2, program feasibility was assessed via participants' feedback toward the training program by three indicators: attendance, future willingness to lead the peer-led self-management program, and leadership skills evaluated by a peer leader training assessment tool.

Results: In this study, peer leaders demonstrated good leadership skills by expressing active willingness to lead self-management programs in the community. Peer leaders' feedback indicated that the program's training content was helpful in preparing peer leaders to guide older adults in learning self-management skills and in improving the abilities and confidence of peer leaders in mentoring self-management.

Conclusion: Findings in this study showed that peer leader training can impact the effectiveness and success of self-management in older adults with diabetes. Even in a small-scale study, the impact was evident, which demonstrated the feasibility of the program. More large-scale studies on the effectiveness of various peer leader training programs in diverse disciplines are recommended.

Clinical Trials Registry: ClinicalTrials.gov Identifier: NCT04298424 (the Peer-Led SelfManagement Program).

Keywords: older adult, peer leader, diabetes, theory-based, peer-led, self-management

\section{Introduction}

Older adults (aged $\geq 65$ years) are at greater risk of diabetes than younger individuals, due to age-related risk factors such as inactivity, obesity, reduced insulin secretion, and dyslipidemia. ${ }^{1,2}$ In older adults with diabetes, disease duration is positively associated with an increased risk of cardiovascular disease. ${ }^{3}$ Furthermore, improper disease 
management and treatment in older adults with diabetes can lead to severe health issues, such as renal lesions and retinopathy, and increase medical costs. ${ }^{4,5}$

The risk of developing diabetes is closely related to unhealthy lifestyles. Positive lifestyle interventions can delay the progression of diabetes in older adults. ${ }^{6}$ To enjoy better quality of life in later life, patients with diabetes must amend harmful lifestyle behaviors, ${ }^{7}$ adhere to diabetes care requirements, and develop the ability to self-manage health issues. ${ }^{8}$ Physical deterioration and various physical and mental factors can, however, significantly increase the challenges of effective disease care and life after contracting diabetes in older adults. ${ }^{9}$ Patients with a lack of confidence in their ability to selfmanage their blood glucose levels and illnesses can develop feelings of frustration and powerlessness, ${ }^{10}$ which further deter efforts to properly adhere to blood glucose self-monitoring, diet, exercise, and other self-management protocols critical for blood glucose control. ${ }^{9}$ Poor implementation of self-management protocols can threaten quality of life, which further affects family life, burdens national health systems and insurance plans, and compromises health promotion efforts in the long run.

Peer leaders with similar cultural backgrounds, languages, socioeconomic statuses, illness experiences, and past successful knowhow in self-management can positively impact their peers' self-management process through improving self-management efficacy, understanding of disease, lifestyle adjustments, ${ }^{11}$ problem-solving skills, and confidence in overcoming barriers. ${ }^{12}$ Peer leaders are trained to promote self-management knowledge, attitude, beliefs, and skills through mutual sharing and emotional support, with the ultimate goal of fostering self-management behaviors in older adults with diabetes. ${ }^{13}$ Peer leaders should demonstrate good communication skills and leadership qualities, which enable them to assist their peers in mastering skills such as self-regulation and problemsolving. ${ }^{14}$

Peer leadership aims to facilitate learning and understanding of health concepts, by drawing on personal experiences and using simple approaches and colloquial diction easily understandable by older adults. Strengthening conceptual reasoning, critical thinking, and problem-solving skills can help improve patient motivation, communication, and questioning skills. ${ }^{14}$ Apposite self-management decisions can then be formed through a combination of personal preferences, values, and needs. Peer leadership is intended to nurture a socially supportive environment that provides targeted support to peers, encourages ongoing participation, improves health behaviors, and achieves successful selfmanagement. At present, most peer leader training (PLT) programs are targeted at adults ${ }^{1,15-17}$ or younger populations. $^{18}$ PLT programs focused on older adults above the age of 65 are scarce. Present PLT programs are typically designed to be executed by healthcare professionals, but healthcare staff can find relating to older adult patients difficult. Thus, peer leaders with successful selfmanagement experiences, similar cultural backgrounds and languages, and related illness experiences are employed to guide and mentor peer patients in the current PLT program. This study aims to improve and promote self-management in older adults with diabetes by developing a communitybased PLT program and assessing its feasibility.

\section{Methods}

This study is the second phase of a large-scale peer-led self-management (PLSM) program. The large-scale program is divided into three phases: the first phase is developing a theory-based PLSM program for older adult peer leaders with diabetes; the second phase is designing a PLT program and assessing its feasibility; the third phase is conducting a large-scale PLSM program for older adults with diabetes in the community. The current study is divided into two stages: stage 1 describes the development of a theory-based PLT program; stage 2 is the assessment of the program's feasibility.

\section{Participant Recruitment and Enrollment}

Inclusion criteria were those aged $\geq 65$ years, with high school or higher level of education, who exhibited leadership traits and communication skills, ${ }^{19}$ and had successful diabetes self-management experience. A total of five peer leaders were recruited.

After recruitment, interviews were conducted to assess the candidates' communication skills and leadership characteristics, and whether they had past successful experience in diabetes self-management. Whether a candidate encompassed leadership characteristics and communication skills was based on recommendations from others or through personal interactions during the recruitment interview. Communication skills were evaluated in terms of their ability to articulate concepts, ideas, emotions, and thoughts. Whether candidates had past successful experience in diabetes self-management was established through comparing glycated hemoglobin, blood pressure, and total cholesterol measures in the past three years, in reference to 
the published standards of the Diabetes Association of the Republic of China (Taiwan). ${ }^{20}$ Participant information such as age, gender, and years living with diabetes were documented via audio recording interviews and collated into Excel for analysis and review.

\section{Ethical Approval and Consent to Participate}

The study was approved by the St. Martin De Porres Hospital (17B-023) Institutional Review Boards in Taiwan, and conducted in accordance with the Declaration of Helsinki. Participants were recruited with help from community-based organizations. Participation was voluntary and all participants gave written, informed consent before interviews, including consent to publication of anonymized responses, and were informed about their right to withdraw at any time.

\section{Stage I: Program Development} Theoretical Framework

Cognitive function and learning ability of older adults are affected by age and disease duration. ${ }^{21,22}$ The peer leader training program in this study was developed based on Kolb's experiential learning theory ${ }^{23}$ as the framework and Bandura's self-regulation theory ${ }^{24}$ as the strategy. The program adopted group learning, with personal experience as the main learning tool. ${ }^{25}$ Peer leaders and peer participants took turns in leading discussions, and sharing of ideas took place in a role-playing fashion, to help enhance effective learning. ${ }^{11,12,23}$

Effective learning is the transformation of life experiences into knowledge, which takes into account various factors, such as the reflective observation of emotions or behaviors. Reflective observations can further form new experiences and bestow different meanings to these experiences. ${ }^{26}$ For instance, through practical implementation of self-management activities and situational case discussions during peer leader training, peer leaders reflect on whether they can identify obstacles in self-management, determine the cause of these obstacles, and contemplate on plans for improvement. The process of transforming experiences, gained from practical implementation and discussions, into new knowledge through reflective observation is known as "abstract conceptualization."

The term "active experimentation" in experiential learning theory entails reflective observation through recognizing similarities and differences between oneself and others' experiences, identifying the cause of a given problem, developing an improvement strategy, and incorporating newfound experiences into future improvement strategies for practical use in subsequent self-management activities or situational case discussions. During situational case discussions, the process of guiding peer participants to reflect on the relationships between daily life habits and blood glucose levels, through observing changes in daily blood glucose levels, is called "self-monitoring," according to the self-regulation theory. ${ }^{24}$ Self-monitoring allows peer leaders to apply new experiences to practical self-management implementation. Only after hands-on execution, are peer leaders able to master learnt skills, which are required for participating in the self-management program.

In addition to reflective observation and self-monitoring of blood glucose, peer leaders observe participant behaviors immediately after self-monitoring, and collate them with personal values and standards, which allows them to define problems, make decisions, and set goals. For instance, case A has a problem of often forgetting to take medication. Through observing the behavior of others (case B \& case $\mathrm{C}$ ), case A can realize his core problems and reflect on his behavior. Such as, case B has the habit of placing his meds on the dining table to remind himself to take pills after meals, while case $\mathrm{C}$ leaves his pills next to the remote control, because he watches television after every meal. Through observing the habits of case $B$ and case $C$, case A can adjust his behavior by placing his medication at a conspicuous spot to remind himself. Peer leaders observe behavioral differences between themselves and other participants, reflect on these disparities, uncover core issues that cause these variances, and come up with apposite improvement strategies through group sharing and discussions. This cognitive process of judging problems, decision-making actions, and setting goals is called self-judgment. ${ }^{27,28}$ Self-judgment enables peer leaders to further consider and examine the differences between this particular behavior and others in self-management. Self-judgment allows peer leaders to deliberate on and compare differences in the selfmanagement behaviors of themselves and others. This process of contemplation and reassessment is equivalent to reflective observation, as conceptualized in experiential learning theory. ${ }^{23}$ The process of formulating and implementing plans for behavioral change involves self-reaction and self-regulation, which is comparable to the abstract conceptualization of experiential learning theory. ${ }^{23}$ Moreover, implementing behavioral change is equivalent 
to active experimentation, as conceptualized in experiential learning theory. ${ }^{23}$

\section{Peer Leader Training (PLT) Program}

The PLT program in this study was a 4-hour weekly session, lasting four consecutive weeks, supplemented by a self-management training manual, designed to reinforce the self-management training skills of peer leaders. Contents of these weekly sessions included: (a) group leading skills and peer leader self-management instructions (including responsibilities of peer leaders and the use and specifications of self-management manuals); (b) researchers led peer leaders in implementing self-management skills through applying experiential learning and self-regulation theories; (c) researchers designed five situational cases that reflected self-management dilemmas commonly encountered in older adults with diabetes. A detailed description of these contents is presented in Table 1. Peer participants were asked to complete home assignments and reflective diary entries after each weekly session. In the event that a peer leader was unable to attend a training session, one-on-one catch-up sessions were held.

\section{Program Components}

\section{Component I: Skills Development}

Skills development was intended to help peer leaders learn self-management skills systematically, lead group discussions on the steps required for mastering core concepts of experiential learning ${ }^{23}$ and self-regulation theories, ${ }^{24}$ and guide older adults with diabetes in learning self-management. This training program aimed to help peer leaders enhance their ability to lead group discussions through applying positive communication skills (for example, listening, encouraging, affirming, sharing experiences, and asking open-ended questions to guide thinking processes). Peer leaders took turns leading four other participants in situational case discussions. Situational case discussions and situational scenarios were used to improve peer leaders' ability to solve problems and overcome obstacles. Additionally, peer participants were guided to implement self-management through applying self-monitoring, selfjudgment, self-reaction, and other strategies, which deepened their familiarity with self-regulation theory and selfmanagement models.

\section{Component 2: Experiential Learning}

Peer leaders acquired and mastered critical self-management skills through experiential learning. First, peer leaders adopted the role of an older adult with diabetes, participated in a 4-week self-management course, and became familiarized with the self-management program. By engaging in self-management activities through role playing, peer leaders perceived possible obstacles and difficulties that peers may encounter, which allowed them to be better prepared to lead groups in the future. Peer leaders observed strategies and techniques used to guide group sharing and discussions, and learned team leadership and self-management training skills. Second, role-simulation exercises in situational scenarios had peer leaders take turns leading other peers in discussions, to practice communication skills, guidance skills, and role functions.

\section{Stage 2: Feasibility Assessment}

A total of five peer leaders were recruited to test the feasibility of this training program. Feasibility of the PLT program was assessed via three indicators and recorded by audio recordings during qualitative interviews. The first indicator was attendance. Peer leaders were required to complete the entire training program to help them effectively master self-management skills. A minimum attendance level of $80 \%$ was required for each weekly session. Successful program completion was defined as a score of $\geq 80$, and at least four peer leaders were expected to achieve this goal. As for the second indicator, researchers employed a peer leader training assessment tool (Table 2) after each weekly session, to assess the ability of peer leaders to apply experiential learning and self-regulation theories properly while conducting program activities, leading situational case discussions, and learning selfmanagement training skills. The peer leader training assessment tool was designed to evaluate the leadership skills of peer leaders. Peer leaders were assessed on their ability to properly guide peer participants in gaining specific experiences and applying reflective observation during role-play simulation and activities. Whether peer participants had effectively learnt these skills determined the leadership skills of peer leaders, and these were evaluated in weekly sessions (Table 2). The third indicator was the willingness of peer leaders, who had successfully completed the PLT program, to promote peer leader selfmanagement programs in the future. This information was obtained by inviting peer leaders to share their thoughts regarding their experiences and participation in the program and offer their opinions on the promotion of PLT programs in the community after completion of training via qualitative interviews. 
Table I Peer Leader Training Program Content

\begin{tabular}{|c|c|c|c|}
\hline Phase & Theme & Aim & Program Content/Activity [Theoretical Basis/Teaching Material] \\
\hline \multirow[t]{2}{*}{1} & $\begin{array}{l}\text { Understands program } \\
\text { model }\end{array}$ & $\begin{array}{l}\text { I. P can identify the } \\
\text { cause of poor } \\
\text { glycemic control (via } \\
\text { self-monitoring of life } \\
\text { events and blood } \\
\text { glucose) }\end{array}$ & $\begin{array}{l}\text { I-I. R gives a self-introduction and explains program content, procedure, and objectives. } \\
\text { I-2. R illustrates group leadership skills. } \\
\text { I-3. R guides } P \text { in making a self-introduction [using the self-management training manual: Icebreaking } \\
\text { Journey] (for example: name, age, number of years living with diabetes, current blood glucose level). } \\
\text { I-4. R guides } P \text { in sharing past experiences and thoughts on self-management using the question-and- } \\
\text { answer method. } \\
\text { I-5. R leads discussion on improvement strategies to address P's self-management difficulties. } \\
\text { I-6. R guides P in discussing Lao-Wu's case [self-management training manual]: "summarize the effects } \\
\text { of life events on Lao Wu's blood glucose control”/“Lao Wu's problem lies in ..." [self-monitoring]. } \\
\text { I-7. R explains details of the self-management plan using Lao Wu's case (see peer leader self- } \\
\text { management plan). }\end{array}$ \\
\hline & & $\begin{array}{l}\text { 2. P can identify } \\
\text { obstacles of self- } \\
\text { management in } \\
\text { situational scenarios } \\
\text { and propose } \\
\text { improvement } \\
\text { strategies }\end{array}$ & 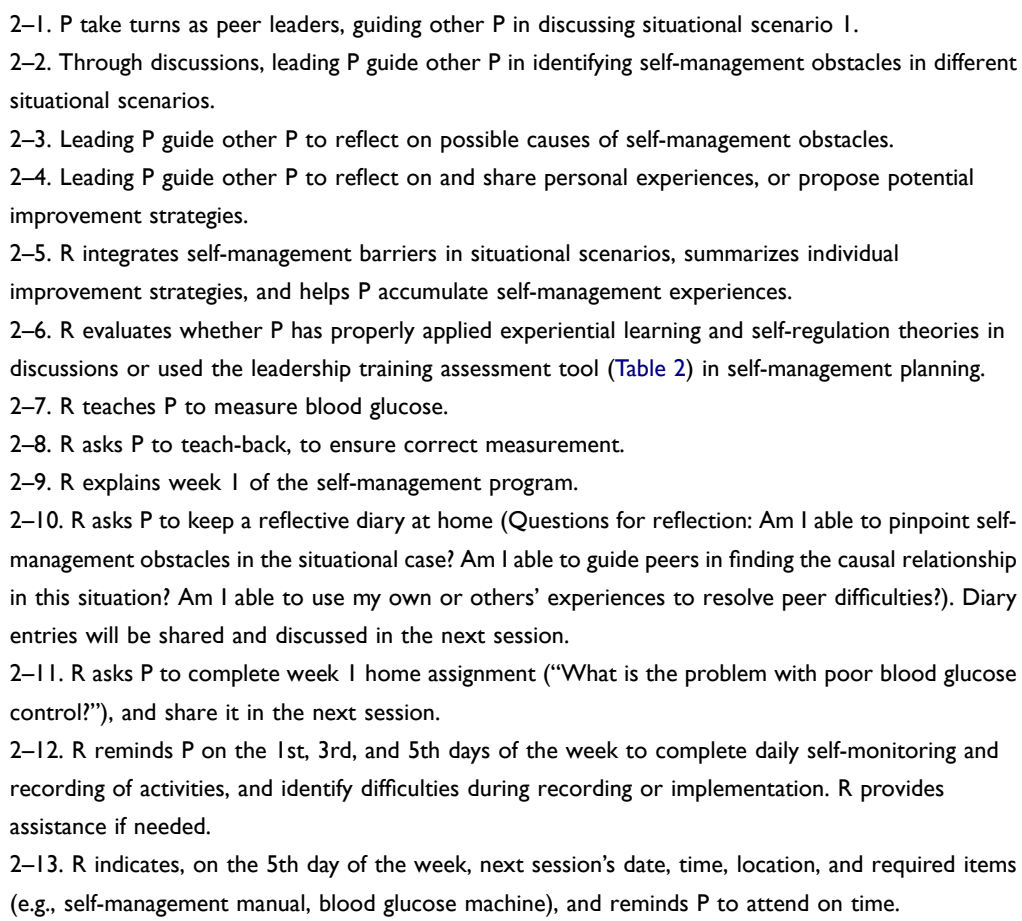 \\
\hline \multirow[t]{2}{*}{2} & & $\begin{array}{l}\text { 1. P can share their } \\
\text { shortcomings in } \\
\text { discussions }\end{array}$ & $\begin{array}{l}I-I . R \text { leads } P \text { in sharing reflective diary and discussion } I . \\
I-2 . R \text { reexamines self-management barriers in the situational scenario, summarizes individual } \\
\text { improvement strategies, and helps } P \text { accumulate self-management experiences. }\end{array}$ \\
\hline & & $\begin{array}{l}\text { 2. P can self-monitor } \\
\text { and record blood } \\
\text { glucose and life } \\
\text { events, identify the } \\
\text { core issues that affect } \\
\text { blood glucose, set } \\
\text { goals, and execute } \\
\text { strategies to address } \\
\text { problems }\end{array}$ & $\begin{array}{l}\text { 2-I. R guides individual } P \text { in sharing week I home assignment. } \\
\text { 2-2. R guides } P \text { in identifying problems and recording contents in home assignments [self-monitoring]. } \\
\text { 2-3. } R \text { and other } P \text { offers advice, to the } P \text { who shared, on glycemic control. } \\
\text { 2-4. } R \text { leads the } P \text { who shared to reflect on how he/she differs from people with well-controlled blood } \\
\text { glucose [self-judgment]. } \\
\text { 2-5. R guides the } P \text { who shared to think about what diabetes problem he/she most wants to resolve. } \\
\text { 2-6. R guides the } P \text { who shared to reflect on what he/she hopes to achieve with the diabetes problem } \\
\text { he/she most wants to resolve. } \\
\text { 2-7. R guides the } P \text { who shared in thinking about what affects target execution [self-judgment]. } \\
\text { 2-8. R guides } P \text { in sharing ways to overcome difficulties. } \\
\text { 2-9. R guides the } P \text { who shared to think about improvement strategies for the diabetes problem that } \\
\text { he/she most wants to resolve [self-reaction]. } \\
\text { 2-10. R collects ideas on how to identify core problems that affect blood glucose changes in daily life } \\
\text { through self-monitoring of blood glucose, observation of differences between those with well- } \\
\text { controlled blood glucose and those without, goal setting, and identification of improvement strategies. }\end{array}$ \\
\hline
\end{tabular}

(Continued) 
Table I (Continued).

\begin{tabular}{|c|c|c|c|}
\hline Phase & Theme & Aim & Program Content/Activity [Theoretical Basis/Teaching Material] \\
\hline & & $\begin{array}{l}\text { 3. P can identify self- } \\
\text { management } \\
\text { obstacles in various } \\
\text { situations and } \\
\text { propose } \\
\text { improvement } \\
\text { strategies }\end{array}$ & 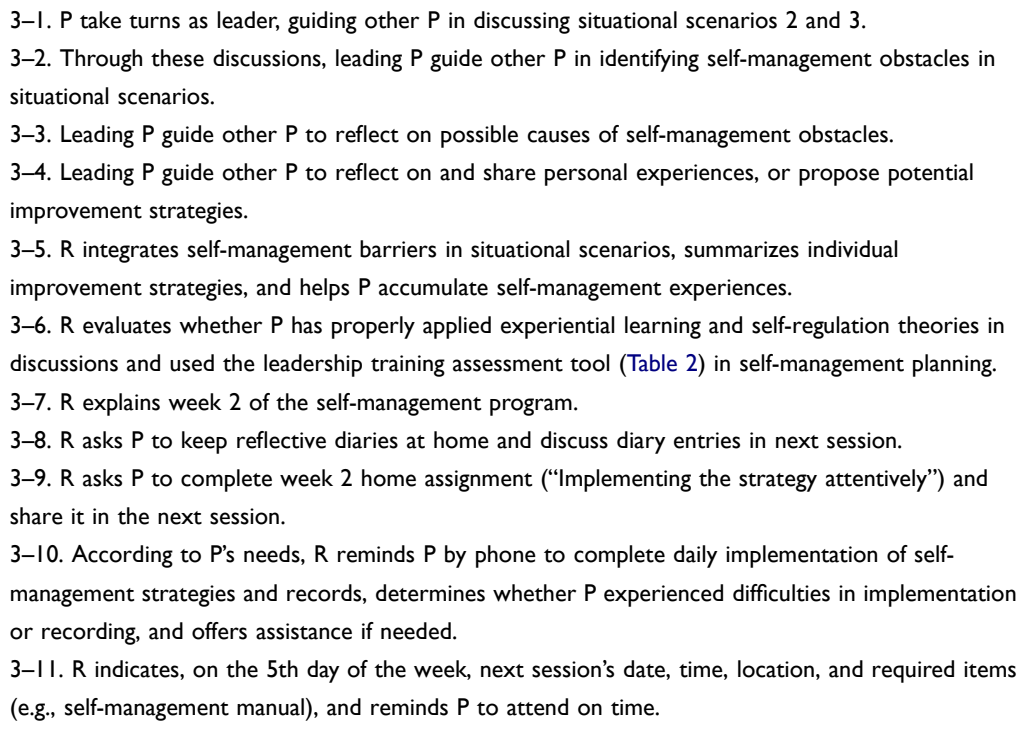 \\
\hline \multirow[t]{3}{*}{3} & & $\begin{array}{l}\text { 1. P can share their } \\
\text { shortcomings in } \\
\text { discussions }\end{array}$ & $\begin{array}{l}I-I . R \text { leads } P \text { in sharing reflective diaries and discussion II. } \\
I-2 . R \text { integrates self-management barriers in situational scenarios, summarizes individual } \\
\text { improvement strategies, and helps } P \text { accumulate self-management experiences. }\end{array}$ \\
\hline & & $\begin{array}{l}\text { 2. P can discuss } \\
\text { difficulties and } \\
\text { successful } \\
\text { experiences address } \\
\text { difficulties, and revise } \\
\text { goals and strategies }\end{array}$ & $\begin{array}{l}\text { 2-I. } R \text { guides individual } P \text { in sharing week } 2 \text { home assignment. } \\
2-2 . R \text { guides the } P \text { who shared in summarizing activities he/she has and has not done and reasons for } \\
\text { it [self-judgment]. } \\
\text { 2-3. } R \text { and other } P \text { offer feedback to the } P \text { who shared. } \\
\text { 2-4. R-guides the } P \text { who shared in modifying goals and practices [self-reaction]. } \\
2-5 . R \text { reexamines reasons for failure, and further re-amends his/her goals, and resets improvement } \\
\text { strategies. }\end{array}$ \\
\hline & & $\begin{array}{l}\text { 3. P can identify self- } \\
\text { management } \\
\text { obstacles in various } \\
\text { situations and } \\
\text { propose } \\
\text { improvement } \\
\text { strategies }\end{array}$ & 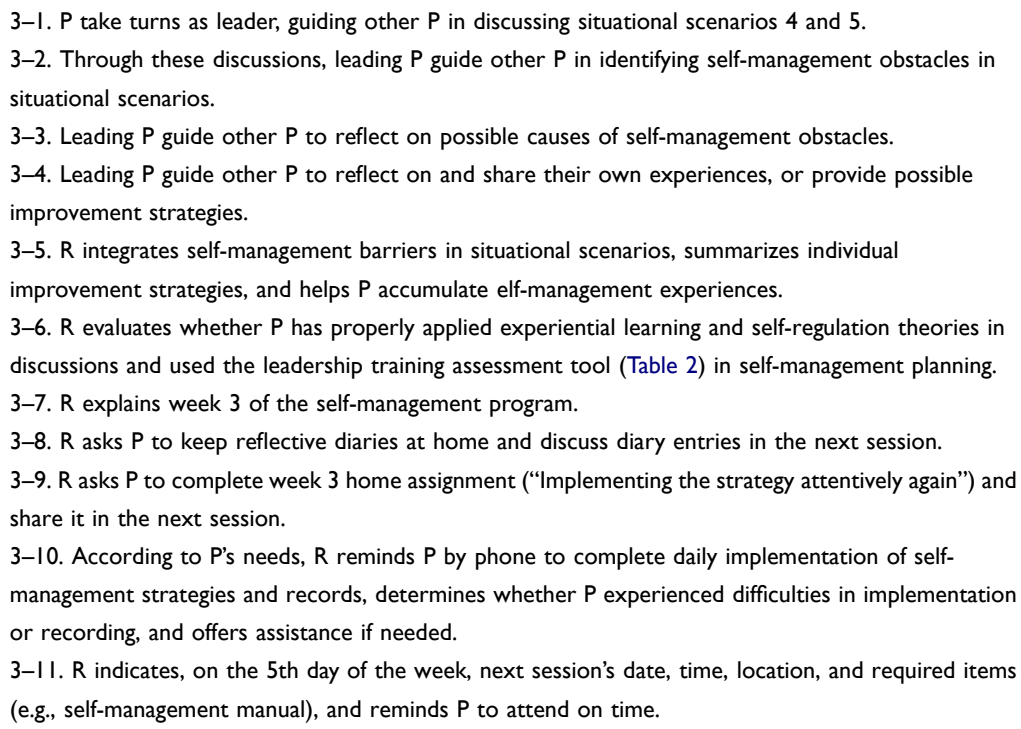 \\
\hline 4 & & $\begin{array}{l}\text { I. P can share their } \\
\text { shortcomings in } \\
\text { discussions }\end{array}$ & $\begin{array}{l}I-I . R \text { leads } P \text { in sharing reflective diaries and discussion III. } \\
I-2 . R \text { integrates self-management barriers in situational scenarios, summarizes individual } \\
\text { improvement strategies, and helps } P \text { accumulate self-management experiences. }\end{array}$ \\
\hline
\end{tabular}

(Continued) 
Table I (Continued).

\begin{tabular}{|c|c|c|c|}
\hline Phase & Theme & Aim & Program Content/Activity [Theoretical Basis/Teaching Material] \\
\hline & & $\begin{array}{l}\text { 2. P can share } \\
\text { implementation } \\
\text { difficulties and } \\
\text { successes, goals, } \\
\text { strategies, reasons } \\
\text { for failure, successful } \\
\text { experiences, and } \\
\text { outcomes }\end{array}$ & $\begin{array}{l}\text { 2-I. } R \text { guides } P \text { in sharing week } 3 \text { home assignment. } \\
\text { 2-2. } R \text { guides } P \text { in summarizing activities they have and have not done and reasons for it [self- } \\
\text { judgment]. } \\
\text { 2-3. } R \text { guides } P \text { in revising new plans to address reasons for failure [self-reaction]. } \\
\text { 2-4. } R \text { guides } P \text { in thinking about the differences before and after lifestyle habit change. } \\
\text { 2-5. } R \text { guides } P \text { in reviewing goals, strategies, reasons for failure, successful experiences, and results of } \\
\text { the course to increase confidence [self-reaction]. } \\
\text { 2-6. Based on P's sharing and reflection, } R \text { summarizes how to systematically lead a 4-week self- } \\
\text { management program. }\end{array}$ \\
\hline & & $\begin{array}{l}\text { 3. } P \text { can use self- } \\
\text { regulation theory to } \\
\text { demonstrate peer } \\
\text { leadership }\end{array}$ & $\begin{array}{l}\text { 3-I. Before the end of the course, } R \text { evaluates whether } P \text { is proficient in using the peer leader training } \\
\text { assessment tool (Table 2). }\end{array}$ \\
\hline
\end{tabular}

Notes: Phase I, concrete experience; Phase 2, concrete experience $\rightarrow$ reflective observation; Phase 3 , concrete experience $\rightarrow$ reflective observation $\rightarrow$ abstract conceptualization; Phase 4, reflective observation $\rightarrow$ abstract conceptualization $\rightarrow$ active experimentation. R, researcher; $P$, peer leaders.

\section{Results}

\section{Participant Characteristics}

Peer leaders who participated in this study were aged 65-75 years, with an average age of 69.2 (standard deviation (SD) 4.3) years. Three (60\%) participants were women and two $(40 \%)$ were men. The mean time since diagnosis of diabetes was 11.8 years (SD 3.8), and $40 \%$ of participants had college or higher degrees.

\section{Program Feasibility}

\section{Achievement of Evaluation Index}

All five peer leaders participated in and completed the entire 4-week training program, yielding an average attendance rate of $100 \%$. At the end of the PLT program, the average assessment score was 88.2 (SD 5.4). All five participants passed the assessment and expressed willingness to promote peer leader self-management programs in the future.

\section{Results of Qualitative Interviews}

After completion of the PLT program, peer leaders shared their thoughts and feedback on their experiences through qualitative interviews. All participants (100\%) concurred that the use of peers with successful self-management experience as trainers was effective for guiding older adults with diabetes (Table 2). Furthermore, all participants $(100 \%)$ concurred that drawing on their own and other peers' experiences in group discussions, to guide self-management learning, helped them better understand self-management concepts.
Qualitative data of the five participants were documented via audio recordings for feasibility assessment. The following presents feedback of participants who played two roles (role of peer leader and role of peer patient). The participants' gender, age, and number of years living with diabetes are noted in parentheses.

\section{Role of Peer Leaders}

Peer leader A (Female, 65, 10 years):

When I was first diagnosed with diabetes, if someone with similar experience had taught me how to take care of myself and told me what to do when I encountered difficulties based on his or her own experience, then I would not have been stuck for so long in my own difficulties.

Peer leader B (Male, 71, 6 years):

These classes let us share our successful experiences in a structured manner. This self-management method is especially good for older patients with diabetes, who can learn from each other's experience and learn more about how to deal with it (their disease)!

Peer leader D (Female, 65, 15 years):

Medical staff teach well, but sometimes I can't understand what they are saying, and I am afraid that I will be embarrassed or laughed at if I don't follow their instructions, so I don't know what to do. It's really good to be taught by my peers.

During training, peer leaders learned to recognize changes and deficiencies in the training process and reflect on ways 
Table 2 Peer Leader Training Assessment Tool

\begin{tabular}{|c|c|c|c|c|}
\hline No. & Item & Score & $\begin{array}{l}\text { Score } \\
\text { Percentage }\end{array}$ & Description \\
\hline I & $\begin{array}{l}\text { Guide participants to gain } \\
\text { specific experiences }\end{array}$ & & 10 & $\begin{array}{l}\text { I. Able to correctly share one's successful diabetes self-management experience ( } 5 \\
\text { points) } \\
\text { 2. Able to correctly guide participants to share the successes, difficulties, and areas } \\
\text { for improvement during implementation of lifestyle changes ( } 5 \text { points) }\end{array}$ \\
\hline 2 & $\begin{array}{l}\text { Guide participants to } \\
\text { self-monitor }\end{array}$ & & 20 & $\begin{array}{l}\text { I. Able to correctly guide participants to keep self-monitoring records using the self- } \\
\text { management manual ( } 10 \text { points) } \\
\text { 2. Able to correctly guide participants to use self-monitoring records to identify core } \\
\text { glycemic control problems ( } 10 \text { points) }\end{array}$ \\
\hline 3 & $\begin{array}{l}\text { Guide participants to } \\
\text { apply } \\
\text { self-judgment }\end{array}$ & & 20 & $\begin{array}{l}\text { 1. Able to correctly guide participants to use self-monitoring records to identify gaps } \\
\text { between their behavior and the ideal or standard behavior ( } 5 \text { points) } \\
\text { 2. Able to correctly guide participants to identify self-management issues ( } 5 \text { points) } \\
\text { 3. Able to correctly motivate participants to improve behavioral problems ( } 5 \text { points) } \\
\text { 4. Able to correctly guide participants to set self-management goals ( } 5 \text { points) }\end{array}$ \\
\hline 4 & $\begin{array}{l}\text { Guide participants to } \\
\text { apply reflective } \\
\text { observation }\end{array}$ & & 10 & $\begin{array}{l}\text { I. Able to correctly guide participants to think about factors that may affect target } \\
\text { execution ( } 5 \text { points) } \\
\text { 2. Able to correctly guide participants to think about possible reasons for failed } \\
\text { execution ( } 5 \text { points) }\end{array}$ \\
\hline 5 & $\begin{array}{l}\text { Guide participants to } \\
\text { apply abstract } \\
\text { conceptualization }\end{array}$ & & 10 & $\begin{array}{l}\text { I. Able to correctly apply their own or peers' successful self-management } \\
\text { experiences, to guide participants in planning self-management strategies ( } 5 \text { points) } \\
\text { 2. Able to correctly guide participants to identify causes of failure, refer to their own } \\
\text { or peers' successful implementation experiences, and revise self-management } \\
\text { strategies ( } 5 \text { points) }\end{array}$ \\
\hline 6 & $\begin{array}{l}\text { Guide participants to } \\
\text { apply self-reaction }\end{array}$ & & 20 & $\begin{array}{l}\text { I. Able to correctly guide participants to set self-management strategies ( } 10 \text { points) } \\
\text { 2. Able to correctly guide participants to implement self-management strategies ( } 10 \\
\text { points) }\end{array}$ \\
\hline 7 & $\begin{array}{l}\text { Guide participants to } \\
\text { apply } \\
\text { active experimentation }\end{array}$ & & 10 & I. Able to guide participants to execute new improvement strategies (I0 points) \\
\hline
\end{tabular}

Notes: Scoring for items 2, 3, and 6: 0 points $=0 \%$ correct, 2 points $=20 \%$ correct, 4 points $=40 \%$ correct, 6 points $=60 \%$ correct, 8 points $=80 \%$ correct, 10 points $=100 \%$ correct. Scoring for items I, 4, 5, and 7: 0 points $=0 \%$ correct, I point $=20 \%$ correct, 2 points $=40 \%$ correct, 3 points $=60 \%$ correct, 4 points $=80 \%$ correct, 5 points $=100 \%$ correct.

to amend these deficiencies. These reflections were further underlined in situational scenarios and weekly home assignments.

Peer leader A:

My blood glucose is well-controlled and my glycosylated hemoglobin maintains below 7\%. However, after monitoring my blood glucose before and after meals, I realized that my understanding of the effects of amount of food on blood glucose was not necessarily accurate. After understanding these measurements, I became aware of the fluctuations in blood glucose levels. In the future, when I lead self-management activities, I will emphasize the importance of self-monitoring to peers.
Peer leader B:

I have had diabetes for many years. Doctors and teachers have told me how I should control blood glucose. However, I have not yet personally experienced disorders due to poorly controlled blood glucose. The sharing and discussion of situational cases and self-management activities enabled me to improve my problem-solving skills through the experiences of others, and I feel more confident leading older patients with diabetes in the future.

Peer leader D:

It's fun when the simulation leader led everyone to share and discuss! This role is played with encouragement and 
openness, and you can't approach sessions with preconceived notions. Watching how peers perform simulations during actual exercises allows me to understand exactly how to do it. This kind of exercise is really good! It makes me more confident.

Peer leader E (Female, 75, 13 years):

In the beginning, I could not immediately identify the obstacles to self-management in the situational case. However, after discussion and analysis, I now know how to make judgments. This learning method is really good!

In situational scenarios, patient's mood was significantly affected by poorly controlled blood glucose levels, which led to complications.

Peer leader A:

Although I am not a psychologist, I can provide psychological support and help find relevant resources to help improve his bad mood.

Peer leader C (Male, 71, 6 years) added, "Availability of resources is really important. We can't be experts in all fields, but I know how to find resources to help him."

\section{Role of Peer Patients}

Peer leaders playing the role of peer patients learnt through situational scenarios.

Peer leader A:

When peer leader $\mathrm{C}$ led the group discussion, I discovered that a leader should grasp the key point of everyone's speech at all times, so he can lead the discussion, and summarize everyone's opinions from the whole discussion in an organized way ... his leadership style is well worth studying!

Peer leader B:

Sometimes we are so keen in giving our own opinions that we neglect time has passed. Peer leader D's leadership style is wonderful. For example, she can always make conclusions instinctively when there is a pause, and bring us back to the core of the discussion. It is really good to learn this practice. I can try it in similar situations in the future.

Peer leader C:

Peer leader A handles silence really well when we don't know what to say. She comes up with topics or questions to lead the discussion. For example: 'What will you do when you feel bad? Who will you meet? Where will you go?' She encourages us to participate in the discussion by sharing her own experiences, which showed me how to lead peers.

Peer leader D:

I have learned a lot from situational discussions and role playing. I feel that peer leaders encounter different situations and I now know how to deal with them when guiding peers. This experience is more real to me than learning though books or talks!

Peer leader E:

I have seen the response of peer leaders $\mathrm{A}$ and $\mathrm{C}$ when they encounter professional medical problems. They seek solution from external resources or help from other peers. For example, asking for other's opinions, or searching for answers through websites. This is important for people like us, who are not medical professionals. With internal and external resources at hand, we are more confident to guide peers.

On the whole, peer leaders' comments demonstrated that they were able to provide assistance through peer support and available resources. Overall, participant response regarding the 4-week training program was positive. General feedback underlined that the training content helped prepare them in mentoring self-management skills of older adults with diabetes and improved their skills and confidence in self-management training.

\section{Discussion}

Using experiential theory as the framework and self-regulation theory as the strategy, older adults with diabetes effectively improved self-management skills through theory-based behavioral and lifestyle changes, such as adopting problem-solving skills systematically; identifying individualized obstacles; and acquiring self-regulation, self-reaction, self-monitoring, self-efficacy, self-judgement, and reflective observational skills. Peer leaders guided peer participants in learning self-management with their knowledge and experience. Peer leaders learnt self-management training skills by continually applying their own experiences and the experiences of peers; reflecting on the relationship and differences between themselves and others in situational scenarios or during self-management implementation; and transforming abstract and obscure ideas into knowledge, through simulation and role-play. These strategies could help build peer leaders' confidence and skills. 
Important insights were gained from the development of this training program. First, researchers recruited older adults with diabetes, who had successful self-management experiences, to participate as peer leaders. Peer leaders, with past successful experiences in diabetes self-management and shared cultural backgrounds and languages with their peers, ${ }^{14}$ were considered eligible to teach diabetes health concepts and problem-solving skills, and to help improve peer self-management motivation and efficacy.

Past peer-led self-management programs typically recruited general patients with diabetes ${ }^{15,16}$ or community workers, ${ }^{11,12}$ without a standardized screening of more eligible candidates as peer leaders. Compared with those with past experiences in self-management, general patients or community workers are usually unfamiliar and inexperienced with diabetes self-management. Thus, more time and effort are needed for them to learn the fundamentals of self-management and acquire the necessary counseling skills. The use of peer leaders who have had past successful experiences in self-management can reduce the time and energy needed for learning basic information (such as on diet, exercise, acute and chronic complications), and shorten the time required for taking fundamental courses. They can thus focus more on the obstacles of patient selfmanagement, to strengthen their counseling skills. Shortened training hours also encourages more peer leaders to participate.

Second, peer leaders with past successful self-management experiences have had similar experiences, so they can better relate to the needs of elderly patients. They can, thus, adequately utilize their past self-management experiences to help peers solve problems, through discussions, sharing, and reflections, which can improve learning. This is consistent with the findings of Kjellsdotter's study of group-based education, ${ }^{26}$ which states that elderly patients with diabetes learn better through a combination of past experiences and experiential learning in diabetes self-management. Thus, in this study, peer leaders adopted experiential learning and self-regulation theories, which are the basis of the PLT program. From these theories, they learned to guide older adults with diabetes in applying daily self-monitoring, identify causes of poor glycemic control, and reflect on the differences between their experiences and those of others or those with ideal behaviors. Peer participants constantly re-assessed whether they could attain desired behavior changes, and further reamend and re-set goals. In this study, researchers had peer leaders draw on examples of their own successful experiences in self-management, to encourage ongoing health behaviors in peers. Successful experiences served as useful references in forming viable self-management behavioral strategies.

In addition, peer leaders took turns role playing as peer leaders and peer participants interchangeably, and rehearsed in five situational scenarios, where common self-management barriers were integrated to simulate the different situations they may encounter. Situational scenarios sought to improve peer participants' leadership qualities and training skills, by encouraging them to share and reflect in group discussions. Moreover, home assignments were assigned for peer leaders to reflect on the implementation process. This helped them transform abstract theoretical concepts into practical, applicable knowledge, which was then reapplied in weekly training sessions. Experiential learning theory as the framework and self-regulation theory as the strategy were used to explain and enable understanding of the learning process and the development of peer leaders. Third, few diabetes-related peer leader training programs that involved the use of multiple teaching methods have been developed. As cognitive decline had always been common in older adults, the use of role-playing and/or simulation (as in simulated self-management program) scenarios helped enhance learning. Role playing was helpful in the learning and practicing of communication skills. Openended questions enabled peer leaders to collect relevant information on an issue. Group sharing and discussions helped identify the most apposite self-management training strategies. Specifically, role playing and simulation created an ideal environment for peer leaders to learn to work in peer-led teams, promote group discussions, and manage group dynamics.

Fourth, although an aim of this training program was to help participants develop good communication skills, such as active listening and reflection, participants whose baseline communication skills were not up to par, based on personal interviews conducted during recruitment, were eliminated from consideration. The two-stage panel and personal interview protocol, used to screen for baseline communication skill in this study, was useful for detection of "danger signals." Many reports on peer leader training programs do not explicitly discuss ways to evaluate communication skills at recruitment.

The program has its limitations. First, peer leaders were required to have at least high school level education, because illiteracy could affect learning outcome. Thus, the effect of this program on peer leaders with less education 
is unknown. Second, peer leaders were required to fulfill good communication and leadership qualifications for inclusion, which excluded otherwise qualified peer leaders with insufficient communication or leadership skills. Third, participants complained that hand-written records were relatively cumbersome for them. In the future, the use of mobile phones, such as LINE app chats and snapshots on mobile cameras, could help facilitate the task.

\section{Conclusion}

Self-management is a critical component of effective diabetes care. Most self-management interventions in older adults with diabetes are led by healthcare professionals, rather than peer leaders, and are focused predominantly on the implementation and evaluation of the intervention itself, rather than on peer leader training, particularly in older adults (aged $\geq 65$ ). To target this problem, peer leaders with successful self-management experiences, similar cultural backgrounds and languages, and related illness experiences were trained to guide and mentor peer patients in this study. Although it is widely recognized that peer leaders can effectively improve diabetes-related health outcomes, few studies have examined the implementation and content of peer leader training programs. On the other hand, of the studies that have reported peer leader training programs, few have provided a comprehensive and detailed description of the training process, which can affect research outcome.

This study aimed to develop and assess the feasibility of a peer leader self-management training program, designed to improve the self-management skills of older adults with diabetes. The results of this study confirmed the feasibility of using peer leaders to conduct self-management training programs. All five participants met the requirements of the primary and secondary feasibility indicators, with a $100 \%$ attendance rate and final evaluation score of $\geq 80$, respectively. Even in a small-scale study, the impact was evident. The findings presented the future potential of this theorybased intervention protocol in improving self-management among older adults with diabetes. The sharing, support, and encouragement among peers had a positive impact on older adults. It was more effective for older adults to learn selfmanagement from peer leaders with similar backgrounds and successful self-management experiences than from healthcare professionals.

This program could serve as a framework to train future peer leaders and as a useful reference in theory testing or in building new knowledge. The feasibility of the peer leader training program could prompt more peer leaders to become future experts in assisting older adults with diabetes to learn self-management, which could help reduce the workload of healthcare professionals. The program could be valuable for researchers or educators to verify actual implementation of the program, which could then serve as a reference to help design and conduct future theory-based intervention studies. A rigorous screening protocol is critical for the identification of appropriate peer leader candidates; thus, it is recommended that researchers develop a comprehensive screening tool to identify potential peer leaders who can be trained as mentors to guide self-management in communities.

\section{Data Sharing Statement}

There is no further data to be shared.

\section{Acknowledgments}

The authors wish to thank the cooperation and assistance of St. Martin De Porres Hospital. We especially thank the peer leaders who participated in this study.

\section{Funding}

This study was made possible through a grant awarded by St. Martin De Porres Hospital research program (P1715).

\section{Disclosure}

To the best of the authors' knowledge, the named authors have no conflict of interest, financial or otherwise.

\section{References}

1. Hajian-Tilaki K, Heidari B, Hajian-Tilaki A. Solitary and combined negative influences of diabetes, obesity, and hypertension on healthrelated quality of life of elderly individuals: a population-based crosssectional study. Diabetes Metab Syndr. 2016;10(2):S37-S42. doi:10.1016/j.dsx.2016.01.018

2. Udenze IC, Taiwo IA, Minari JB, Adeyemo WL. Insulin resistance and cardiovascular risk in older adult Nigerians with type 2 diabetes. Int $J$ Noncommunicable Dis. 2019;4(1):21-26. doi:10.4103/jncd. jncd_43_18

3. Sattar N, Rawshani A, Franzén S, et al. Age at diagnosis of type 2 diabetes mellitus and associations with cardiovascular and mortality risks: findings from the Swedish national diabetes registry. Circulation. 2019;139(19):2228-2237. doi:10.1161/CIRCULATION AHA.118.037885

4. Kash BA, Lin SH, Baek J, Ohsfeldt RL. The diabetes management education program in south Texas: an economic and clinical impact analysis. Front Public Health. 2017;5:345. doi:10.3389/ fpubh.2017.00345

5. Cho S, Kim M, Park K. Self-management levels of diet and metabolic risk factors according to disease duration in patients with type 2 diabetes. Nutr Res Pract. 2018;12(1):69-77. doi:10.4162/ nrp.2018.12.1.69 
6. Cheng AYY. Introduction. Canadian diabetes association clinical practice guidelines expert committee. Clinical practice guidelines. Can J Diabetes. 2013;37:S1-3. doi:10.1016/j.jcjd.2013.01.009

7. Blackford K, Jancey J, Lee AH, et al. A randomised controlled trial of a physical activity and nutrition program targeting middle-aged adults at risk of metabolic syndrome in a disadvantaged rural community. BMC Public Health. 2015;15(1):284. doi:10.1186/s12889015-1613-9

8. Brenk-Franz K, Strauss B, Tiesler F, et al. The influence of adult attachment on patient self-management in primary care - the need for a personalized approach and patient-centred care. PLoS One. 1991;50 (2):e0136723. doi:10.1016/0749-5978(91)90022-L

9. Werfalli MM, Kalula SZ, Manning K, Levitt NS. Does social support effect knowledge and diabetes self-management practices in older persons with type 2 diabetes attending primary care clinics in Cape Town, South Africa? PLoS One. 2020;15(3):e0230173. doi:10.1371/ journal.pone.0230173

10. Luque-Reca O, Augusto-Landa JM, Pulido-Martos M. Emotional intelligence and depressive symptoms in Spanish institutionalized elders: does emotional self-efficacy act as a mediator? Peer J. 2016;4:e2246. doi:10.7717/peerj.2246

11. Debussche X, Besançon S, Balcou-Debussche M, et al. Structured peer-led diabetes self-management and support in a low-income country: the ST2EP randomised controlled trial in Mali. PLoS One. 2018;13(1):e0191262. doi:10.1371/journal.pone.0191262

12. Cade JE, Kirk SFL, Nelson P, et al. Can peer educators influence healthy eating in people with diabetes? Results of a randomized controlled trial. Diabet Med. 2019;26(10):1048-1054. doi:10.1111/ j.1464-5491.2009.02808

13. Kaven MH, Hesampour M, Ghahremani L, Tabatabaee HR. The effects of a peer-led training program on female students' self-esteem in public secondary schools in Shiraz. J Adv Med Educ Prof. 2014;2 (2):63-70.

14. Snyder JJ, Wiles JR. Peer led team learning in introductory biology: effects on peer leader critical thinking skills. PLoS One. 2015;10(1): e0115084. doi:10.1371/journal.pone.0115084

15. Tang TS, Funnell MM, Gillard M, Nwankwo R, Heisler M. Training peers to provide ongoing diabetes self-management support (DSMS): results from a pilot study. Patient Educ Couns. 2011;85(2):160-168. doi:10.1016/j.pec.2010.12.013

16. Tang TS, Funnell MM, Gillard M, Nwankwo R, Heisler M. The development of a pilot training program for peer leaders in diabetes. Diabetes Educ. 2011;37(1):67-77. doi:10.1177/ 0145721710387308
17. McCallum GB, Chang AB, Wilson CA, et al. Feasibility of a peer-led asthma and smoking prevention project in Australian schools with high indigenous youth. Front Pediatr. 2017;5:Article 33. doi: $10.3389 /$ fped 2017.00033

18. Sebire SJ, Edwards MJ, Campbell R, et al. Protocol for a feasibility cluster randomised controlled trial of a peer-led school-based intervention to increase the physical activity of adolescent girls (PLAN-A). Pilot Feasibility Stud. 2016;2(1):2. doi:10.1186/s40814-015-0045-8

19. Stenov V, Wind G, Vallis M, Reventlow S, Hempler NF. Groupbased, person-centered diabetes self-management education: healthcare professionals' implementation of new approaches. BMC Health Serv Res. 2019;19(1):368. doi:10.1186/s12913-019-4183-1

20. Chen JF, Chen $\mathrm{HS}$, Chu $\mathrm{CH}$, et al. Executive summary of the DAROC clinical practice guidelines for diabetes care-2018. Diabetes association of the Republic of China (Taiwan). J Formos Med Assoc. 2020;119(2):577-586. doi:10.1016/j.jfma.2019.02.016

21. Arcos-Burgos M, Lopera F, Sepulveda-Falla D, Mastronardi C. Neural plasticity during aging. Neural Plast. 2019;2019:1-3. doi:10.1155/2019/6042132

22. Zhou J, Zhang Z, Zhou H, Qian G. Diabetic cognitive dysfunction: from bench to clinic. Curr Med Chem. 2019;26. doi:10.2174/ 1871530319666190206225635.

23. Kolb DA. Experiential Learning: Experience as the Source of Learning and Development. Prentice-Hall; 2014:c1984.

24. Bandura A. Social cognitive theory of self-regulation. Organ Behav Hum Decis Process. 1991;50(2):248-287. doi:10.1016/0749-5978 (91)90022-L

25. Yao TY, Tseng CH. A study on the motivation and change process of middle-aged people participating in community service learning: a case of Kaohsiung City. Bull Chung Hwa Univ Med Technol. 2018;48:85-103.

26. Kjellsdotter A, Berglund M, Jebens E, Kvick J, Andersson S. To take charge of one's life-group-based education for patients with type 2 diabetes in primary care-a lifeworld approach. Int J Qual Stud Health Well-Being. 2020;15(1):1726856. doi:10.1080/17482631.2020.1726856

27. Clark NM, Janz NK, Dodge JA, Sharpe PA. Self-regulation of health behavior: the "take PRIDE" program. Health Educ Q. 1992;19 (3):341-354. doi:10.1177/109019819201900306

28. Tougas ME, Hayden JA, McGrath PJ, Huguet A, Rozario S, Courvoisier DS. A systematic review exploring the social cognitive theory of self-regulation as a framework for chronic health condition interventions. PLoS One. 2015;10(8):e0134977. doi:10.1371/journal. pone. 0134977
Journal of Multidisciplinary Healthcare

\section{Publish your work in this journal}

The Journal of Multidisciplinary Healthcare is an international, peerreviewed open-access journal that aims to represent and publish research in healthcare areas delivered by practitioners of different disciplines. This includes studies and reviews conducted by multidisciplinary teams as well as research which evaluates the results or conduct of such teams or healthcare processes in general. The journal covers a very wide range of areas and welcomes submissions from practitioners at all levels, from all over the world. The manuscript management system is completely online and includes a very quick and fair peer-review system. Visit http://www.dovepress.com/testimonials. php to read real quotes from published authors. 